\title{
THE INFLUENCE OF ENVIRONMENTAL CONDITIONS OVER ADAPTIVE CROSSOVER BASED ON THE DISTRIBUTION OF CHROMOSOMES IN SEARCH SPACE
}

\author{
Rares MANIU ${ }^{1}$ \\ ${ }^{1}$ Ph.D. (c), Military Technical Academy, 39-49 George Cosbuc Bvd, Bucharest, Romania \\ rares.maniu@yahoo.com
}

\begin{abstract}
The adaptability of genetic algorithms is given by adjustment of capacity of their genetic operators by controlling their operating parameters. Optimal control of these parameters is a challenge to evolutionary computation. The aim is both to increase the performance and to obtain an evolutionary algorithm that does not require data provided by the operator and operation specific for each problem. The present paper is a study of the influence of environmental conditions (different types of graphs to the problem of finding the shortest path) over adaptive crossover based on the distribution of population of chromosomes in search space. We will demonstrate that a direct dependent parameter from position of chromosome in search space, in combination with direct dependent parameter from fitness values, ensure a degree of adaptability of crossover operator compared with adaptive genetic operator who takes into consideration only the value of fitness function.
\end{abstract}

\section{INTRODUCTION}

The balance between crossover and mutation probability is critical to the success of genetic search. The optimal rate between these is different for different problems and for different stages of genetic search. If the mutation assures the diversity of chromosomes in solutions space, being a divergent operator, the crossover assures the convergence of genetic algorithm, being a convergent operator.

Traditionally, the parameters of genetic algorithms were found using trial-and-error method. Later, researchers are focused to develop methods for adapting genetic operators, the main purpose being to develop a full auto adapted genetic algorithm.

In [1], the probability of mutation is variable during optimization run. In [2], authors used a genetic algorithm with a variable mutation operator based on the dependence of the mutation probability upon two parameters, the dimension of the search space and the population size.

In [3], authors developed a mechanism for adapting crossover and mutation operators. In this case, during the execution of genetic algorithm, the crossover and mutation are adapted according to the population maximum and mean fitness.

Robert Hinterding, in [4], developed the idea of multichromosome, Using multichromosomes, he encoded different aspects of given problem onto distinct chromosomes. This kind of representation performs better then a classic genetic algorithm. The idea to use multichromosomes to separate the main and complex problem in a number of simple problems was used by Juliff in [5] or by Chow in [6].
In all of these studies, the main variables that are used for adaptation are environmental conditions and the results generated by genetic algorithm. These variables are used to modify internal parameters of genetic search.

\section{RELATED WORK}

In almost all cases, the results produced by a genetic algorithm are evaluated using fitness parameter. But, another indication for convergence is the grouping of chromosomes in a limited region of search space, where is placed the optimum. Thus, if we want to develop an adaptive operator, is a good idea to take in consideration both fitness value and the placement of chromosomes in the search space as a measure of diversity.

If for fitness exists some parameters to evaluate the convergence (maximum, minimum, medium fitness values), it is necessary to exist a measure to evaluate the placement of chromosomes as a measure of diversity. This measure can be the central chromosome, proposed by Maniu in [7] or "moment of inertia" proposed by Morrison and DeJong in [8].

According to [7], the central chromosome is defined as:

$$
m_{i}=\left[m_{i}(0), m_{i}(1), \ldots, m_{i}(n)\right] \text {, }
$$

Where:

$$
m_{i}(k)=\sum_{j=0}^{N} X_{j}(k) / N .
$$

$\mathrm{N}$ is the number of chromosomes in generation and $x(k)$ is the $k$-th allele of the chromosome. To evaluate the distance between chromosomes, according to [7], it is calculated like this: 


$$
\operatorname{dist}\left(X_{k}, y_{k}\right)=\sum_{i=0}^{N}\left[X_{k}(n)-y_{k}(n)\right]
$$

Where $X_{k}, y_{k}$ chromosomes in k-generation, $\mathrm{N}$ represents the length of chromosomes and $X_{k}(n), y_{k}(n)$ are the $n$ allele from chromosomes $\mathrm{x}, \mathrm{y}$.

Sure, the central chromosome is not necessary to be a valid solution for problem; it is in fact a measure that indicates the movement of chromosomes in search space.

Using this measure, in [9] is developed an adaptive probability of crossover. This probability is defined by:

$$
p_{c}=a \frac{f_{\max }-f_{\text {current }}}{f_{\max }-\bar{f}} * \frac{\operatorname{dist}\left(X_{\text {current }}-X_{\text {central }}\right)}{\operatorname{dist}\left(X_{\max }-X_{\text {central }}\right)}
$$

Where:

- $\quad a$ is an integer parameter,

- $X_{\max }$ is chromosome with the best fitness from generation,

- $X_{\text {central }}$ is the central chromosome,

- $X_{\text {current }}$ is the current chromosome,

- $f_{\text {current }}$ is the fitness of current chromosome,

- $f_{\max }$ is the maximum fitness in generation,

- $\bar{f}$ is the average fitness of all chromosomes in generation.

In this equation, the parameter $\frac{\operatorname{dist}\left(\boldsymbol{X}_{\text {current }}-\boldsymbol{X}_{\text {central }}\right)}{\operatorname{dist}\left(\boldsymbol{X}_{\max }-\boldsymbol{X}_{\text {central }}\right)}$ is direct dependent from position of chromosome in search space and $\frac{f_{\max }-f_{\text {current }}}{f_{\max }-\bar{f}}$ is a dependent parameter from fitness values.

Using this crossover probability, in [7] is demonstrated that the use of adaptive genetic operators instead of classic, fixed genetic operators in genetic algorithms gives them a clearly superior operation.

This paper is a study of the influence of environmental condition over a genetic algorithm that used an adaptive crossover with probability defined by equation (3). These results are compared with the results produced by a genetic algorithm that use a crossover operator without a dependent parameter from position of chromosome in search space, described by $p_{\text {c-onlfititess }}=\frac{f_{\max }-f_{\text {current }}}{f_{\max }-\bar{f}}$.

The adaptive crossover will be tested with different types of graphs (different number of nodes and links) and with different values for $a$ parameter used in equation no.3.

\section{TESTS AND RESULTS}

The genetic algorithm implemented for tests used a population of 30 chromosomes, for parent's generation and for offspring and for next generation.

The mutation operator is classic, randomly a chromosome being replaced with another. This new chromosome is randomly generated. The mutation rate is $5 \%$.

Adaptive crossover operator uses equation no (3) to exchange genetic information. Depending on the probability of crossover is replaced a variable part of best adapted chromosome from the two chosen, being a single point cut with variable position.

Tests were performed on two graphs, each with 80 nodes. The first graph has 1500 edges and the second 2500 edges.

For each of the two cases was studied the influence of a parameter from equation no. (3), test values being $0,0.5,1,1.5,2,2.5,3$ and 10 . The results are shown in the following figures:

\section{a. A graph with 80 nodes and 1500 edges:}

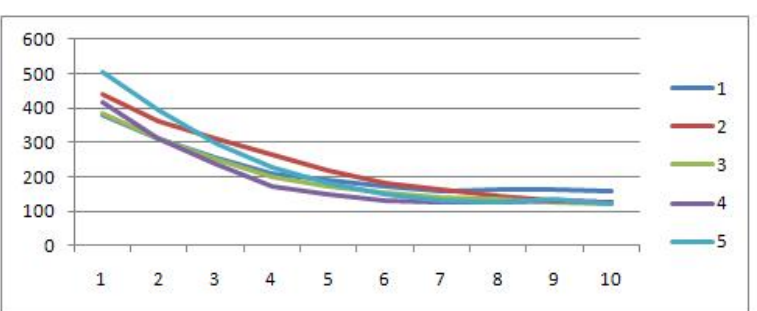

Figure 1 Fitness values obtained with crossover without direct dependent parameter from position of chromosome in search space

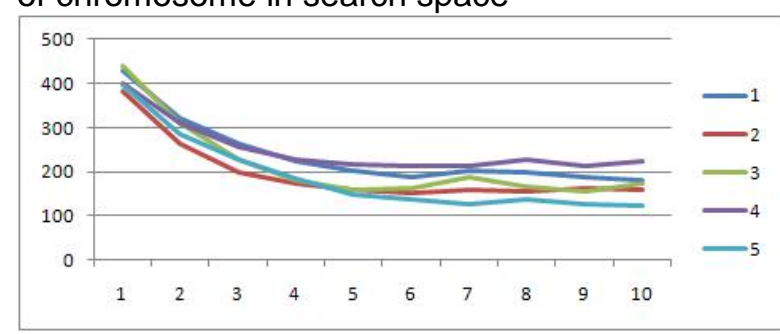

Figure 2 Fitness values obtained with adaptive crossover, $a=0$ 


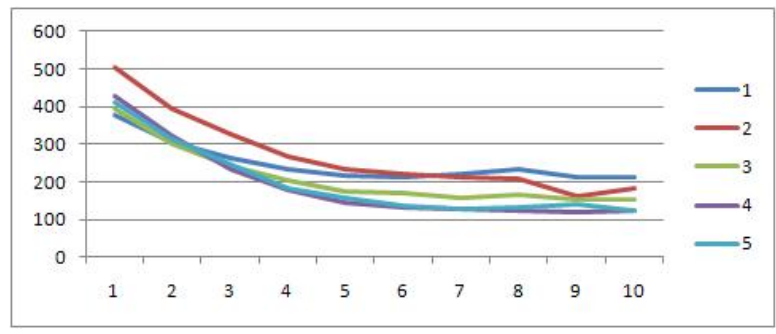

Figure 3 Fitness values obtained with adaptive crossover, $a=0.5$

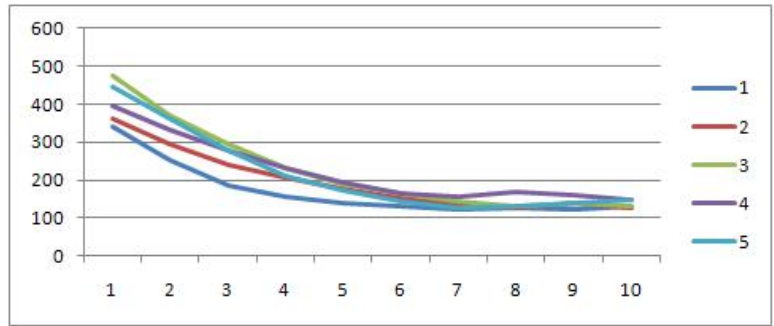

Figure 4 Fitness values obtained with adaptive crossover, $a=1$

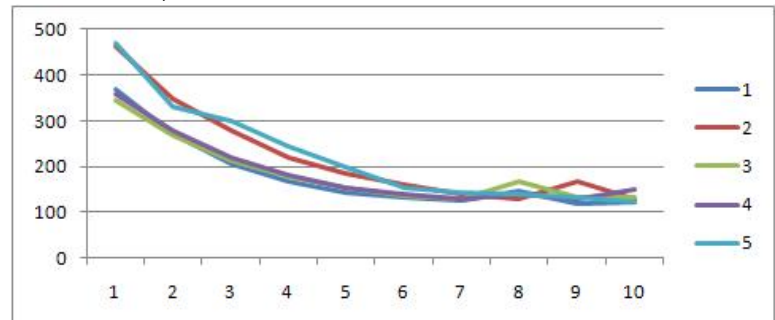

Figure 5 Fitness values obtained with adaptive crossover, $\mathrm{a}=1.5$

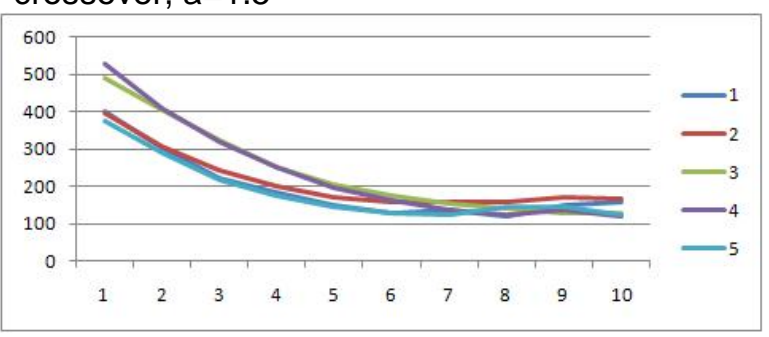

Figure 6 Fitness values obtained with adaptive crossover, $\mathrm{a}=2$

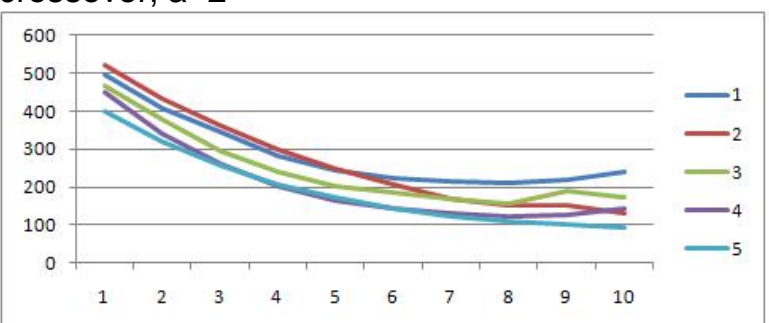

Figure 7 Fitness values obtained with adaptive crossover, $\mathrm{a}=2.5$

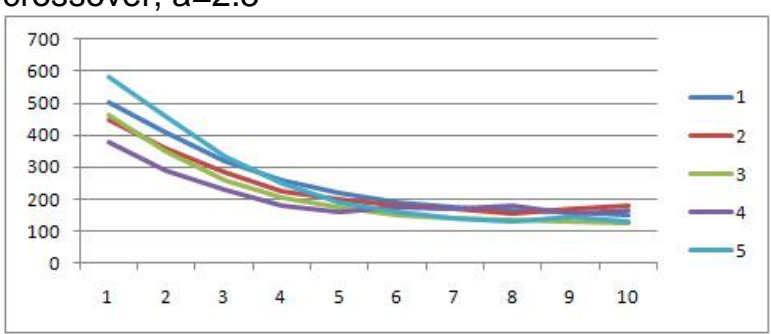

DOI: 10.21279/1454-864X-17-11-075

(c) 2017. This work is licensed under the Creative Commons Attribution-Noncommercial-Share Alike 4.0 License.
Figure 8 Fitness values obtained with adaptive crossover, $\mathrm{a}=3$

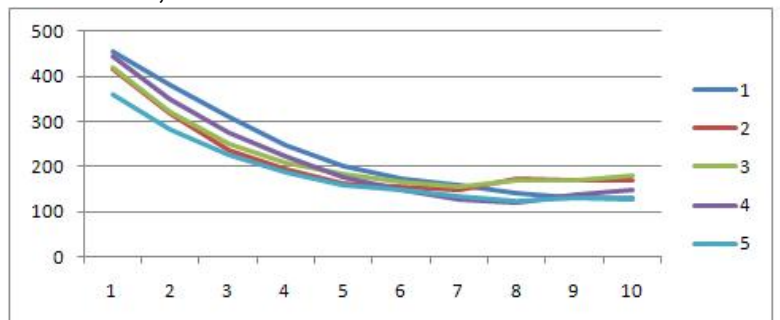

Figure 9 Fitness values obtained with adaptive crossover, $a=10$

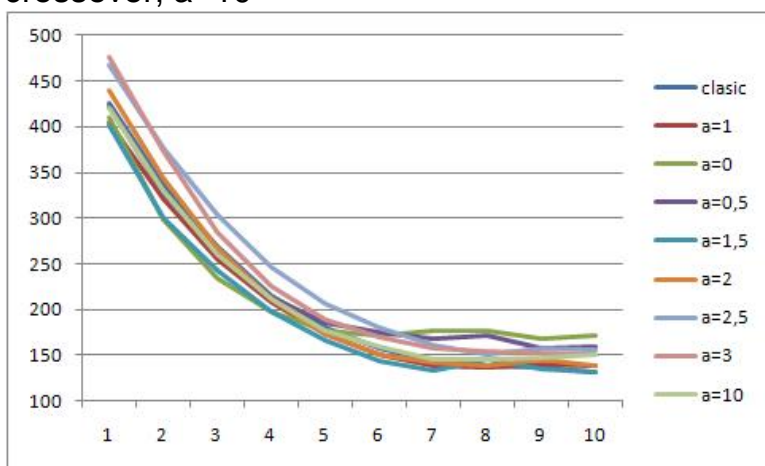

Figure 10 Medium fitness values obtained with adaptive (with different values for a parameter) and classical crossover

b. A graph with 80 nodes and 2500 edges

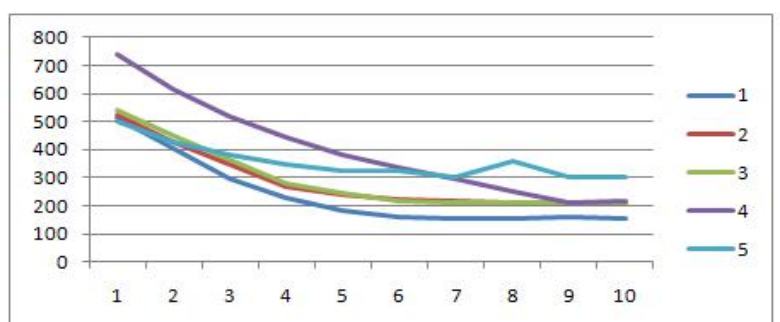

Figure 11 Fitness values obtained with crossover without direct dependent parameter from position of chromosome in search space

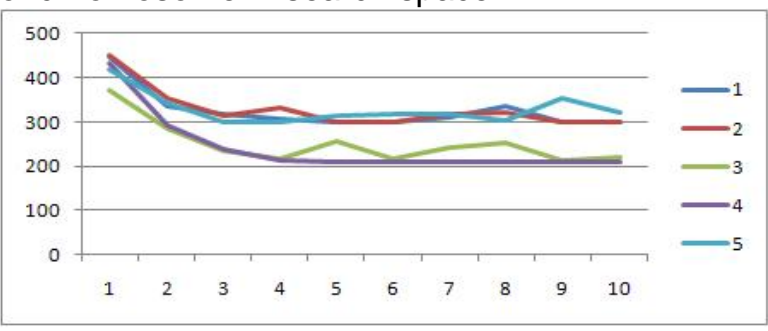

Figure 12 Fitness values obtained with adaptive crossover, $\mathrm{a}=0$

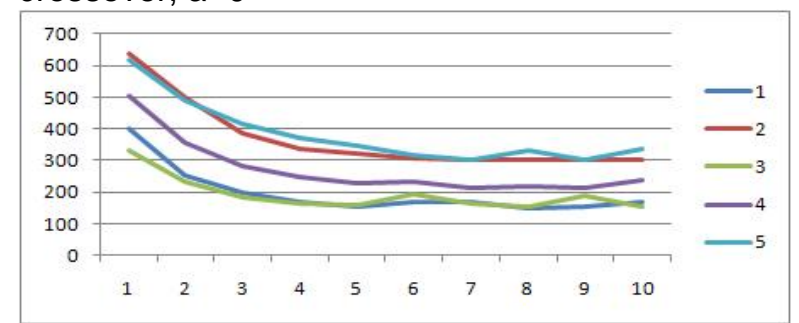


Figure 13 Fitness values obtained with adaptive crossover, $\mathrm{a}=0.5$

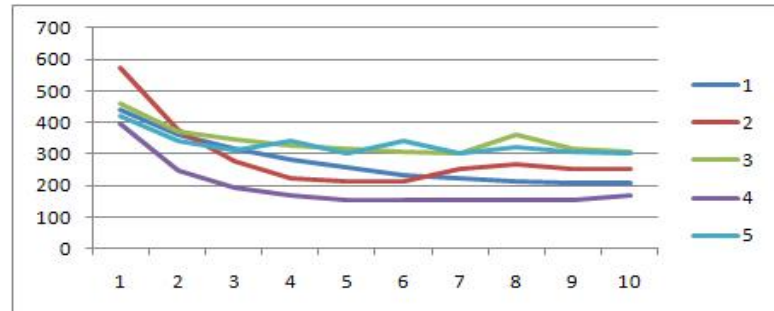

Figure 14 Fitness values obtained with adaptive crossover, $\mathrm{a}=1$

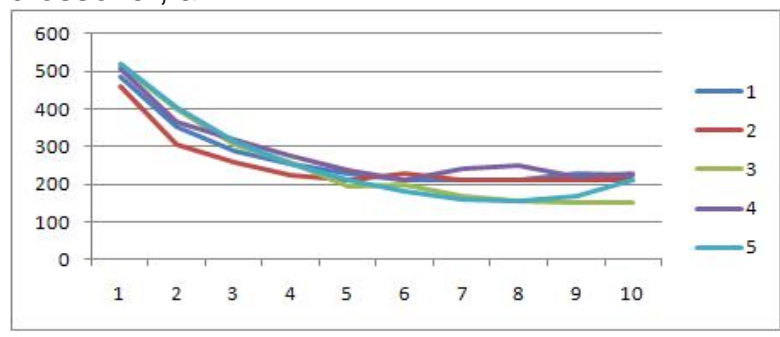

Figure 15 Fitness values obtained with adaptive crossover, $\mathrm{a}=1.5$

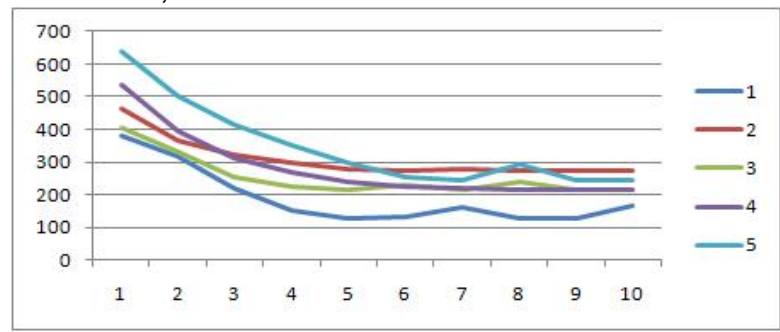

Figure 16 Fitness values obtained with adaptive crossover, $\mathrm{a}=2$

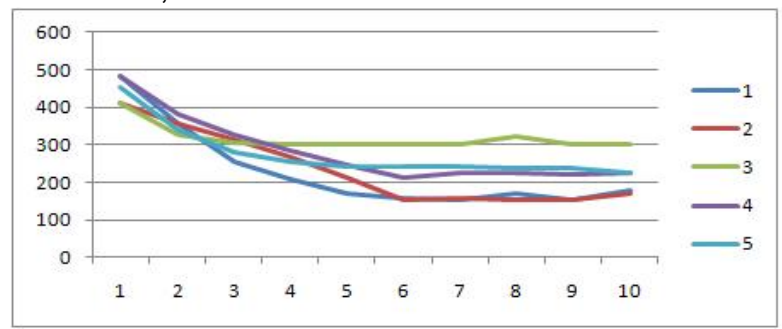

Figure 17 Fitness values obtained with adaptive crossover, $\mathrm{a}=2.5$

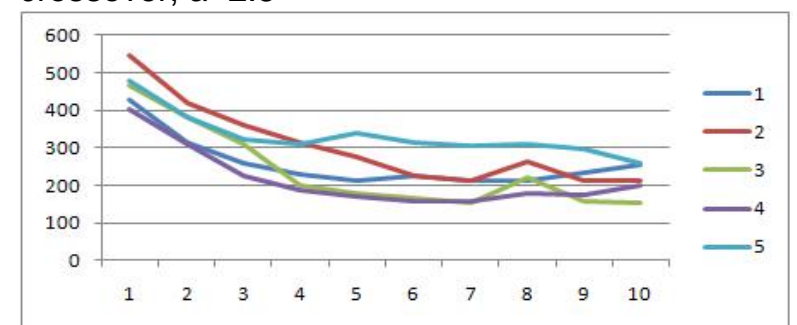

Figure 18 Fitness values obtained with adaptive crossover, $\mathrm{a}=3$

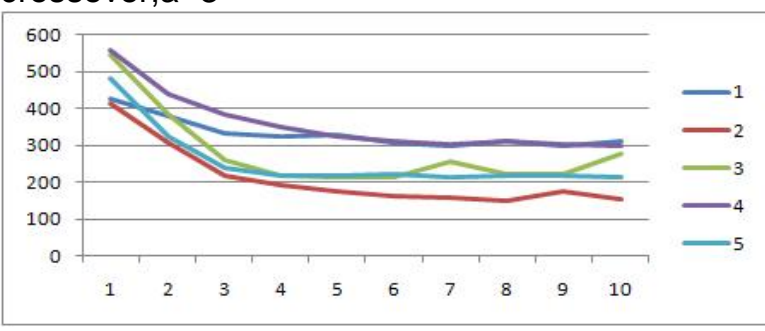

Figure 19 Fitness values obtained with adaptive crossover, $a=10$

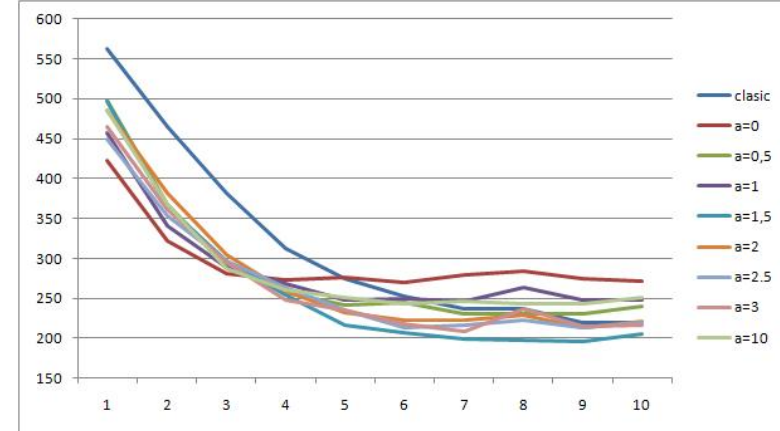

Figure 20 Medium fitness values obtained with adaptive (with different values for a parameter) and classical crossover

\section{Conclusions}

In all cases, the algorithm evolves towards the state of convergence, all graphs showing the declining value of fitness over these ten generations.

From the results obtained for the tested values of a parameter, we can see a large dispersion value $a=0$. This is normal, because $a=0$ means that the probability $p_{c}=0$. The result is total replacement of the fittest chromosome crossover. In this case the adaptive crossover operator becomes no longer functional. With increasing value of this parameter, the dispersion obtained for values of fitness is decreasing, the minimum dispersion obtaining to the interval $(1,1.5)$, both for less dense graphs(1500 edges) and more dense graphs(2500 edges). Also around these values for a parameter, it is obtained minimum values of average fitness, at the end of the ten run of the algorithm.

The increasing of the value for a parameter produce the increase of the probability of crossover $P_{c}$ and a substantially reducing of exchange of genetic material between two chromosomes that undergo the operation of crossover. This is reflected in an increasing of dispersion for fitness values as noticed in previous figures. 
A high value of a parameter $(a=10)$ produce a minimum exchange of genetic material, the algorithm becoming a simple random search in solutions space.

Analyzing the average values of fitness for both two types of adaptive crossover, it can be observed that the decrease is steeper for adaptive crossover with the parameter dependent from position of chromosome in search space than for crossover without it. In all cases, the algorithm tends to convergence. The minimum of fitness values for adaptive crossover is obtained if a parameter is in $(1,1.5)$ interval.

Considering these observations, we conclude that, both for less dense graphs and for graphs with a higher density of edges, the adaptability of operator crossover with direct dependent parameter from position of chromosome in search space offers better solutions.

Also, the value for a parameter included in the probability of crossover $p_{c}$ must be in the interval $(1,1.5)$ to obtain a correct function of adaptive crossover that use a probability described in equation (3).

\section{Future developments}

As shown in tests, the presence of a term dependent on the placement of chromosomes in search space in crossover operator leads to improvement of the search. The results are influenced by the a parameter of the equation no. 3. A correctly chosen value of this parameter will improve the convergence and produce a more predictable response of the algorithm.

In this study, it is observed that there is a range of values for a parameter that lead to improve the search results. To achieve an adaptive crossover is necessary to develop a mechanism for establishing automatic of this parameter for each issue separately.

Also as a further development, the position of chromosomes in search space can be considered for changing the size of populations of chromosomes during genetic search, with direct effect on the speed of algorithm.

\section{Bibliography}

[1] Fogarty,T.C.Varying The probability of mutation in the genetic algorithm. Proceedings of the 3rd International Conference on Genetic Algorithms, 1989, pp.104-109.

[2] Hesser J., Männer R. Towards an optimal mutation probability for genetic algorithms. In: Schwefel HP., Männer R. (eds) Parallel Problem Solving from Nature. PPSN 1990. Lecture Notes in Computer Science, vol 496. Springer, Berlin, Heidelberg

[3] M. Srinivas and L. M. Patnaik, Adaptive probabilities of crossover and mutation in genetic algorithms in IEEE Transactions on Systems, Man, and Cybernetics, vol. 24, no. 4, pp. 656-667, Apr 1994

[4] R. Hinterding, Self-adaptation using multi-chromosomes Evolutionary Computation, 1997, IEEE International Conference on, Indianapolis, IN, 1997, pp. 87-91

[5] K. Juliff, A multi-chromosome genetic algorithm for pallet loading. Proceedings of the 5th International Conference on Genetic Algorithms, pp. 467-473, 1993.

[6] Chow R. Evolving Genotype to Phenotype Mappings with a Multiple-Chromosome Genetic Algorithm. In: Deb K. (eds) Genetic and Evolutionary Computation - GECCO 2004, pp. 1006-1017 Springer, Berlin, Heidelberg

[7] R. Maniu, Adaptive mutation in genetic algorithms for shortest path routing problem 7th International Conference on Electronics, Computers and Artificial Intelligence (ECAI), Bucharest, 2015, pp. S-69-S-74..

[8] R.W. Morrison, K.A. De Jong, Measurement of Population Diversity In: International Conference on Artificial Evolution (Evolution Artificielle), pp.31-41, 2002, Springer, Berlin, Heidelberg

[9] R. Maniu, L.A. Dumitru, Genetic Algorithm - Adaptive Crossover Based on Solution Distribution in Search Space, OPTIM-ACEMP 2017, Brasov, Romania 